\title{
The nosology of involuntary movements in older people
}

\author{
Jolyon Meara and Peter Hobson \\ University of Wales College of Medicine, Rhyl, UK
}

\section{Editorial}

Involuntary movements become increasingly common with age and often lead to considerable disability, handicap and social embarrassment. ${ }^{1}$ Many causes of involuntary movements can be readily treated once the correct diagnosis is established. Getting the diagnosis right in older people is often challenging even for specialists. Even the identification of conditions thought easy to classify in younger people, such as Parkinson's disease, can be very difficult in older adults. ${ }^{2}$ This burden of movement disorder in older people reflects the increasing prevalence of neurodegenerative and vascular disease with age as well as the growing exposure to medication and the natural history of conditions such as essential tremor, which tend to worsen with age and precipitate medical presentation later in life. A complex and poorly understood relationship exists between motor control, disorders of mood and cognitive function. In older subjects involuntary movements are often associated with gait abnormalities and poor mobility and falls may be the presenting feature.

\section{Epidemiology}

Several community-based studies have indicated that involuntary movements are common in older people and are often undiagnosed and therefore untreated. ${ }^{1,3-4}$ The age-specific prevalence of the commonest causes of involuntary movements -essential tremor, Parkinson's disease and druginduced movement disorders increase dramatically with age. The epidemiology of chorea and ballism has been little studied and the few published studies of dystonia suggest that adult-onset cases tend to be focal rather than generalized. Blepharospasm

Address for correspondence: J Meara, University Dept. of Geriatric Medicine, Glan Clwyd Hospital, Rhyl, Denbighshire LL18 5UJ, UK.

is an interesting form of dystonia that appears to occur in older women and usually is associated with a degree of oromandibular/craniocervical dystonia.

The large Europarkinson collaborative study reported a prevalence of $2.3 \%$ for parkinsonism and $1.6 \%$ for Parkinson's disease in a sample of over fourteen thousand subjects over the age of 65 years age-adjusted to a standard population. ${ }^{5}$ A study of a retirement community, Carefree, Arizona revealed that, out of 356 subjects over 65 years of age, $43 \%$ had tremor, $20 \%$ could be diagnosed as having essential tremor and $7 \%$ had Parkinson's disease. ${ }^{1}$ Nearly two-thirds of the PD group in this study had not been previously diagnosed. Door-to-door total census studies of PD suggest that at least a third of subjects with PD are medically undetected. Minimal signs of extrapyramidal dysfunction are increasingly described in a high proportion of older people but their significance is still unclear, though may be related to cognitive decline and increased mortality. ${ }^{6}$ Whether subtle signs of extrapyramidal dysfunction reflect subclinical striatal Lewy body disease or striatal Alzheimer type pathology is not known.

Drug-induced movement disorders in older people require further attention. Studies of parkinsonism have tended to exclude subjects known to have been exposed to neuroleptic drugs, in order to minimize problems of diagnostic accuracy. However, reports suggest that around $10-20 \%$ of prevalent cases of parkinsonism may be due to neuroleptic exposure. ${ }^{3,7}$ The risk of tardive dyskinesia is known to be strongly associated with increasing age and female sex. It is still unclear how common spontaneous non-drug related orofacial dyskinesia is in older people. Orofacial dyskinesia, developing many years after tooth extraction and the non-wearing of dentures, is well recognized. Elderly subjects in nursing and 
residential homes have a particularly high prevalence of movement disorders and are at high risk of being overlooked. ${ }^{8}$

\section{Diagnostic accuracy}

The accurate diagnosis of movement disorders in older people is often challenging. Co-morbidity, cognitive impairment, multiple drug exposure, poor tolerance of trials of drug treatment and the lack of diagnostic tests considerably reduce accuracy in older subjects. ${ }^{9}$ Drug-induced movement disorders are common, yet often poorly appreciated by the prescriber, and accurate drug histories are often difficult to obtain.

The use of clinical diagnostic criteria that have been developed for a range of movement disorders can improve diagnostic accuracy. Inevitably all such tools offer a compromise between sensitivity and specificity. The UK PDS Brain Bank clinical diagnostic criteria for Parkinson's disease in the hands of experts have been shown to improve accuracy. ${ }^{10}$ However, clinical diagnostic criteria in Parkinson's disease may have approached their maximal utility, due to the existence of very atypical forms of true Parkinson's disease. Outside of expert hands, the situation still remains far less satisfactory, with high levels of misdiagnosis and inappropriate treatment with antiparkinsonian drugs. ${ }^{9}$ The challenge of differentiating between essential and parkinsonian tremor still remains. How can diagnostic accuracy be improved? Clearly elderly patients should be referred earlyon for specialist assessment and may often require a period of observation before the correct diagnosis, based on validated clinical criteria, becomes apparent. Geriatricians with an interest in movement disorders also probably make far less use than neurologists of video recordings as a diagnostic aid. The use of DaTSCAN, a radioligand that binds to the presynaptic dopamine transporter, may help with the classification of atypical postural tremors in older people, by confirming the functional status of the nigrostriatal tract. ${ }^{11}$ Late-onset Huntington's disease is becoming increasingly recognized in older subjects and needs to be considered as a cause of late-onset chorea. The impact of psychogenic involuntary movements in older people has not been specifically addressed.

\section{Treatment}

Many involuntary movements can respond to treatment. Sometimes this involves no more than stopping an offending drug. Prochlorperazine $\left(\right.$ Stemetil $^{\mathrm{TM}}$ ) and metclopramide (Motilium $^{\mathrm{TM}}$ ) prescribed to older people for dizzy spells and stomach upsets, along with antipsychotic medication, still continue to keep movement disorder clinics in steady business. In Parkinson's disease, data are emerging suggesting that disease progression may be modified by some forms of early drug treatment, thus making early detection and diagnosis all the more important. Apomorphine remains an under-used treatment for older patients with Parkinson's disease, and the effects of intermittent injection and continuous infusion need to be evaluated formally in older patients to demonstrate the role of this drug in disease management.

The new treatment modality of botulinum toxin can make a significant impact on the quality of life of older patients with tremor, dystonia (particularly blepharospasm), and other involuntary movement. Botulinum toxin will be valuable in dystonic conditions as anticholinergic drugs are very poorly tolerated by older people. In selected older patients, deep brain stimulation is also beginning to offer the hope of much more effective treatment for essential tremor, Parkinson's disease and tremor associated with multiple sclerosis.

\section{References}

1 Khatter AS, Kurth MC, Brewer MA et al. Prevalence of tremor and Parkinson's disease. Parkinson's Related Disord 1996; 2: 205-08.

2 Meara RJ, Bhowmick BK, Hobson JP. Accuracy of diagnosis in patients with presumed Parkinson's disease in a community-based disease register. Age Ageing 1999; 28: 99-102.

3 Morgante L, Rocca WA, Di Rosa AE et al. Prevalence of Parkinson's disease and other types of parkinsonism: a door-to-door survey in three Sicilian municipalities. Neurology 1992; 42: 1901-07.

4 Wang S-J, Fuh J-L, Teng EL et al. A door-to-door survey of Parkinson's disease in a Chinese population in Kinmen. Arch Neurol 1996; 53: 66-71.

5 de Rijk MC, Tzourio C, Breteler MMB et al. Prevalence of parkinsonism and Parkinson's disease in Europe: the EUROPARKINSON collaborative study. J Neurology Neurosurg Psychiatry 1997; 62: 10-15. 
6 Bennett DA, Beckett LA, Murray AM et al. Prevalence of parkinsonian signs and associated mortality in a community population of older people. N Eng J Med 1996; 334: 71-76.

7 Mutch WJ, Dingwall-Fordyce I, Downie AW et al. Parkinson's disease in a Scottish city. BMJ 1986; 292: 534-36.

8 Moghal S, Rajput AH, Meleth R et al. Prevalence of movement disorders in institutionalised elderly. Neuroepidemiology 1995; 14: 297-300.

9 Meara J, Bhowmick BK. Parkinson's disease and parkinsonism. In: Meara J, Koller WC eds.
Parkinson's disease and parkinsonism in the elderly. Cambridge: Cambridge University Press, 2000.

10 Hughes AJ, Daniel SE, Kilford L et al. Accuracy of clinical diagnosis of idiopathic Parkinson's disease: a clinicopathological study of 100 cases. $J$ Neurol Neurosurg Psychiatry 1992; 55: 181-84.

11 Benamer H, Patterson J, Grosset D et al. Accurate differentiation of parkinsonism and essential tremor using visual assessment of $\left[{ }^{123} \mathrm{I}\right]$ FP-CIT SPECT imaging. Mov Disord 2000; 15: 503-10. 